\title{
Image Segmentation Adapted for Clinical Settings by Combining Pattern Classification and Level Sets
}

\author{
S. Li, T. Fevens, and A. Krzyżak \\ Computer Science Department, Concordia University, Montréal, Québec, Canada \\ \{shuo_li, fevens, krzyzak\}@es.concordia.ca
}

\begin{abstract}
An efficient clinical image segmentation framework is proposed by combining a pattern classifier, hierarchical and coupled level sets. The framework has two stages: training and segmentation. During training, first, representative images are segmented using hierarchical level set. Then the results are used to train a pattern classifier. During segmentation, first the image is classified by the trained classifier, and then coupled level set functions are used to further segment to get correct boundaries. The classifier provides an initial contour which is close to correct boundary for coupled level sets. This speeds up the convergence of coupled level sets. A hybrid coupled level set method which combines minimal variance functional and Laplacian edge detector is proposed. Experimental results show that by the proposed framework, we achieve accurate boundaries, with much faster convergence. This robust autonomous framework works efficiently in a clinical setting where there are limited types of medical images.
\end{abstract}

Keywords: Segmentation, variational level set, support vector machine, variational methods, energy minimization, Bayesian decision.

\section{Introduction}

Image segmentation has always been a critical component in medical imagery since it assists in medical diagnoses. It is more challenging compared to other imaging fields. This is primarily due to the large variability in topologies, complexity of medical structures, several kinds of artifacts and restrictive scanning methods. This is especially true for volumetric medical images where a large amount of data is coupled with complicated 3D medical structures.

One of latest techniques in segmentation is based on the class of deformable models, referred as "level set" or "geodesic active contours/surfaces." The application of the level set in medical image segmentation became extremely popular because of its ability to capture the topology of shapes in medical imagery. There are some related works that have been proposed mainly for two-dimensional segmentation. Codimension-two geodesic active contours were used in [10] for the segmentation of tubular structures. The fast marching algorithm [11] and level set method were used in [7] and [14], while Region competition, introduced in [17], was used in [2]. In [2] and[15], Chan and Vese proposed a method using the Mumford-Shah model for both 
two dimensional and volumetric segmentation. Later a hierarchical scheme was used to extend this method to segment multiple regions [14]. Very recently Michal et al [6] proposed a hierarchical volumetric segmentation which combines Chan and Vese method and geodesic active contour [1] with edge alignment for volumetric segmentation. To apply level set methods to real time applications, LeFohn [8] has translated level set techniques to graphic cards and run in nearly real time.

Although efficient, level sets are not suitable for clinical segmentation due to several reasons: (1) high computational cost; (2) complicated parameter settings; (3) sensitivity to the placement of initial contours. With regard to the latter, as will be shown in experimental results, the running time of level set method heavily relies on the position and size of initial curves and complexity of objects. Moreover for some cases, coupled level set do not converge for some initial curves. This paper reports the efforts to overcome the above problems by combining pattern classifier and level sets as a continuation of work reported in [9]. The approach takes the strength of classifier, hierarchical and coupled level set segmentation. In this framework, clinical segmentation is divided into three steps. First, sample images or representative slices of volumetric images are segmented by the hierarchical level set segmentation method. The hierarchical method allows the detection of multiple objects in an image while limiting the complexity of the computation. Then these results are used to train the pattern classifier. We choose support vector machine (SVM) which is widely used in pattern recognition. Therefore the segmentation problem is expressed as a classification problem temporarily. Since medical images usually contain a large amount of redundant information for classification, to accelerate the segmentation by SVM, an information reduction scheme [9] is used. Although SVM training takes some time, once the SVM is trained, we can use it to segment the image whenever we want. Finally, coupled level sets are used to represent multiple objects. The evolution of these level set curves will give a final fine segmentation. A hybrid coupled level sets segmentation algorithm combining Samson's algorithm [12], optimal edge integrator [7] and geodesic active contour model is also proposed. Although the second stage only gives a coarse result, it helps the coupled level set curves by finding good initial curves. Therefore it takes much less time for the coupled curves to converge. By above framework, with the help of SVM, we naturally combined hierarchical and coupled level set to achieve a fast and robust autonomous medical image segmentation framework for clinical setting. An error analysis model is also proposed to analyze and compare hierarchical and coupled level sets.

\section{Segmentation Methods: Variational Approach}

Minimal Variance Functional. Due to its simplicity and efficiency, minimal variance is widely used in variational level set methods [2, 12 and 15]. In our framework, two minimal variance based level set methods will be combined. A brief introduction is given below. To focus our discussion, regularity terms will be ignored. In [2], Chan and Vese proposed a variational level set approach based on minimal variance and 
Mumford-Shah functional described as following. Given a two-dimensional gray level image, $I(x, y): \Omega \rightarrow R^{+}$.

$$
E\left(C, c_{1}, c_{2}\right)=\iint_{\Omega_{c}}\left(I-c_{1}\right)^{2} d x d y+\iint_{\Omega_{1} \Omega_{c}}\left(I-c_{2}\right)^{2} d x d y,
$$

where $C$ is the active contour which separates the image into interior and exterior parts respectively. And $c_{1}$ and $c_{2}$ are the means of interior and exterior parts of $C$.

Samon [12] proposed a similar minimal variance functional for coupled level sets. Assume there are $K$ regions; the proposed method used $K$ level set functions $\phi_{i}(i$ $\in[1, k])$ to represent each of them as

$$
E_{\text {coupled }}\left(\phi_{1}, \ldots, \phi_{k}\right)=\sum_{i=1}^{K} e_{i} \int_{\Omega} H_{\alpha}\left(\phi_{i}\right)\left(I-c_{i}\right)^{2} / \sigma_{i}^{2} d x d y, \forall i, \alpha_{i} \in \mathbb{R} .
$$

where $H_{\alpha}$ is the Heaviside function, $c_{i}$ and $\sigma_{i}$ are mean and covariance of positive areas in level set function $\phi_{i}$.

Hierarchical versus coupled. Level set methods naturally divide an image into two regions. Therefore it is very efficient at extracting one object in an image consisting of several disconnected pieces. In order to extract multiple objects, people normally use hierarchical methods [5, 12] or coupled level set methods [15, 16, 17]. Although straightforward and fast, hierarchical segmentations simply assume a single mean inside and outside of zero level. So for multiple objects detection, it tends to be less accurate than coupled level sets. As a result the boundaries may not be optimal. A detailed error analysis is performed and shown in section 2. Coupled level set on the other hand uses one level set function to represent each object. But it is not only slow but it also suffered from the problem of placement of initial curve, a common problem that exists in numerical minimization when functions are non-convex the numerical results may depend on the choice of the initial curves $[6,15]$.

Error Analysis with Bayes model. Although hierarchical level set segmentation is widely used in segmentation, the accuracy of the obtained boundaries is unknown. In the following, a Bayes model is proposed to analysis the results. A simulation on energy minimization for hierarchical and coupled level set is performed and compared. In this simulation, data is randomly generated with given means and covariance. We use Bayes decision to calculate the correct decision boundary and benchmark with the decision boundary calculated by the energy minimization. The error measurements are defined in eq. 3. The correct decision boundary is calculated using Bayesian decision approach.

Fig. 1(a) shows that when there are two classes in the image, energy minimization is able to get perfect decision boundary which is almost the same as Bayesian decision boundary. However when there are more than two classes, hierarchical scheme will introduce more error as shown in Fig. 1(b). Moreover the energy curve in Fig. 1 (b) is not very sharp around the global minima which means that numerical algorithm may not converge to global minimum. Fig. 2 shows energy surface of eq. 3 . The error is much lower than with hierarchical level sets. From above we can con- 
clude that coupled level sets tend to find more accurate decision boundaries than those for hierarchical level sets when there are more than two classes in the image.

1. $E_{c}\left(w_{1}, w_{2}\right)=\int_{0}^{w 1}\left(I-\mu_{\text {left }}\right) d x+\int_{w \mathbf{1}}^{w 2}\left(I-\mu_{\text {middle }}\right) d x+\int_{w \mathbf{2}}^{\text {max }}\left(I-\mu_{\text {right }}\right) d x$.

$E_{h}(w)=\int_{0}^{w}\left(I-\mu_{\text {left }}\right) d x+\int_{w}^{\max }\left(I-\mu_{\text {right }}\right) d x, w_{i}$ are decision boundaries.

2. Normalized energy $E(x)=E(x) / N$ where $N$ is the total number of pixels.

3. $e_{1}=$ misclassed pixels/total pixels;

$e_{2}=$ misclassed pixels/pixels of two classes

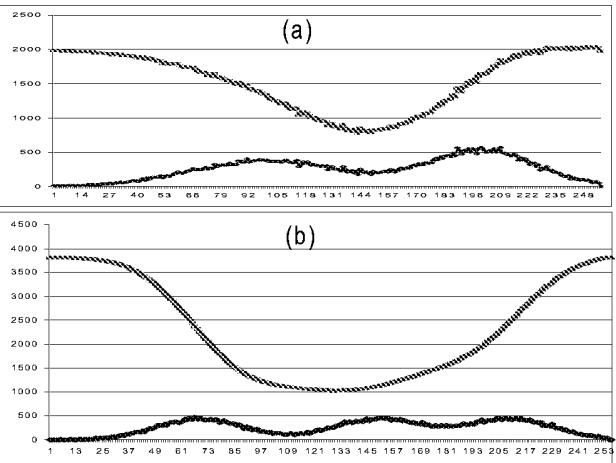

Fig. 1. Energy minimization and Bayesian boundary. Top curve: energy curve $E_{h}$ or $E_{c}$. Bottom curve: histogram of the image. (a) Two regions case: first decision boundary by hierarchical energy minimization $\left(e_{1}=0.0020 ; e_{2}=0.0020\right)$. (b) Three regions case: first decision boundary calculated by hierarchical energy minimization $\left(e_{1}=0.0607 ; e_{2}=0.0911\right)$.

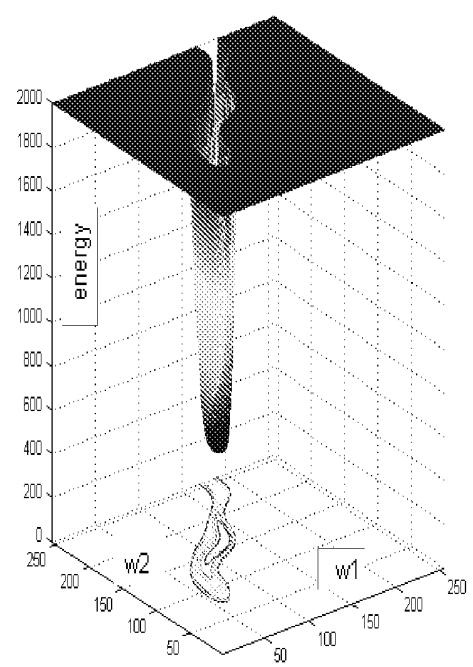

Fig. 2. Energy minimization and Bayesian boundary using energy function $E_{c}\left(e_{1}=0.0207 ; \quad e_{2}=0.0411\right)$.

\section{Proposed Framework}

The proposed framework has two stages: a training stage and a segmentation stage. In the training stage, first, representative images are segmented using hierarchical level set region detection. Then the results are used to train a SVM classifier. In segmentation stage, first images are classified by the trained SVM, and then coupled level set functions are used to further segment the images to get accurate boundaries.

Hierarchical region detection. First hierarchical level set described in section 2.1 is used to detect hierarchically how many regions inside of representative images. The energy function we use is shown in eq. 1. Level set formula is given in [2]. 
Optimal SVM training and segmentation. Due to the fact that level set normally takes long time to segment the image, to adapt it to clinical applications, recently researchers $[9,4]$ are working on initializing a level set segmentation with an approximated segmentation from another method to get a more time-efficient method. In [4], Chen et al. initialize level set with marching cube method. In [9], Li et al. initialize level set with a trained SVM in volumetric image and clinical images. In current application, we adapt the SVM based initialization proposed in [11, 12].

The results obtained from hierarchical region detection are input into SVM. A window based feature extraction is used to extract features from the regions segmented by hierarchical level set segmentation. The SVM classifier we use is modified from [3]. To accelerate the segmentation by SVM, an information reduction is used [9]. For segmentation we only need to compute $3 \%$ of the total pixels since rest of the $97 \%$ are the repetition of the $3 \%$.

Hybrid Coupled Level Sets Segmentation. SVM classification naturally provides a novel initial contour for coupled level sets. We use one level set function to represent one region. Since boundary segmented by SVM is close to the correct boundary, a hybrid coupled level sets segmentation algorithm combining Samson's algorithm [12], optimal edge integrator [7] and geodesic active contour model are proposed as shown in eq. 4. A similar functional is used in [1] for a single level set function.

$$
E=-\gamma_{1} E_{\text {Edge }}+\gamma_{2} E_{\text {Coupled }}+\gamma_{3} E_{G A C},
$$

where $\gamma_{i}$ are constants and geodesic active contour $\left(E_{G A C}\right)$ is defined as $E_{G A C}=\iint g(S) d S$ where $g(S)$ is an inverse edge indicator function. As suggested in [1], we use $g(S)=$ $\alpha^{2} /\left(\alpha^{2}+|\nabla I|^{2}\right)$, where $\alpha$ is a constant. $E_{E d g e}$ is the functional proposed in [7] where Kimmel shows that the Laplacian edge detector $\Delta I$ provides optimal edge integration with regard to a very natural geometric functional as shown in eq. 5 .

$$
E_{\text {Edge }}(S)=\iint_{S}\langle\nabla I, \mathbf{n}\rangle d a-\iint_{\Omega_{S}} K_{I}|\nabla I| d x d y,
$$

where $S$ is the evolving contour, $K_{I}$ is the mean curvature of level sets, $\boldsymbol{n}$ is the unit vector to the curve and $d a$ is the surface area element.

Eq.6 shows the level set function we use. In eq.6, $\phi: \Omega \rightarrow R^{+}$is Lipschitz continuous function, $\delta_{\alpha}$ is the direct delta function, $I_{\xi \xi}=\Delta I-K_{I}|\nabla I|$ and $\beta$ is a constant.

$$
\frac{\phi_{i}^{t+1}-\phi_{i}^{t}}{\Delta t \cdot \delta_{\alpha}\left(\phi_{i}^{t}\right)}=\left[\gamma_{3} \operatorname{div}\left(g \frac{\nabla \phi_{i}}{\left|\nabla \phi_{i}\right|}\right)-\gamma_{2} e_{i} \frac{\left(u_{0}-c_{i}\right)^{2}}{\sigma^{2}}-\beta \frac{\left(\sum_{i=1}^{K} H_{\alpha}\left(\phi_{i}\right)-1\right)^{2}}{\left(u_{0}-c_{i}\right)^{2}}-\gamma_{1} I_{\xi \xi}\right] \text {. }
$$

Confidence map. To measure the confidence of the segmentation, a confidence measurement is proposed: $\psi(x, y)=\max \left(H_{a}\left(\phi_{i}\right) / \Sigma\left|H_{\alpha}\left(\phi_{i}\right)\right|\right)$. The larger the value of $\psi(x, y)$, the higher the confidence of the data segmented. 


\section{Experiments}

First, some experiments were done to show the final results and computational time of the level set in terms of the number of iterations to convergence could heavily rely on position and size of initial curve and complexity of the object. The level set algorithm is described in section 3.1. Then different two-dimensional and volumetric images are used to test the framework we proposed.
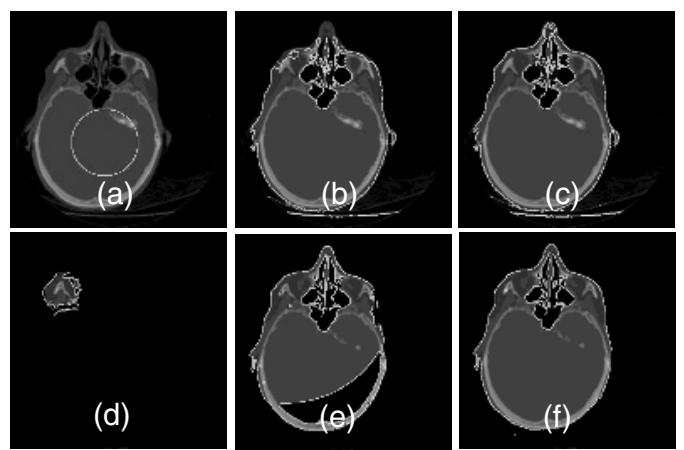

Fig. 3. Challenging nose. (a) Iteration 0. (b) Iteration 150. (c) Iteration 350. (d) Iteration 0. (e) Iteration 600. (f) Iteration 1200.
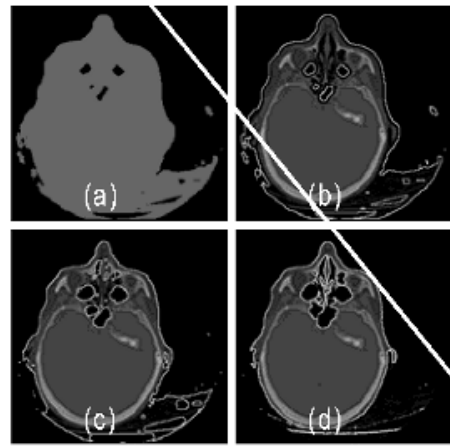

Fig. 4. Comparison of level set (b, c, and d) and SVM based segmentation (a). (a) SVM based. (b) Iteration 0. (c) Iteration 5. (d) Iteration 15.
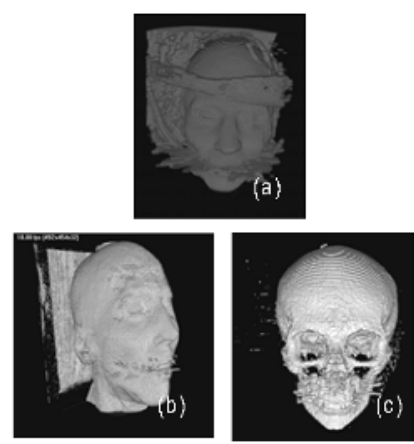

Fig. 5. Volumetric segmentation results. (a) Volumetric segmentation by SVM. (b)

Volumetric segmentation by hierarchical level set. (c) Internal part (skull) rendering.

As can be seen from Figs. 3 (a) to (c), the majority of the objects are segmented in the first 150 iterations while it takes another 200 iterations to segment only the nose. It is very difficult for the user to set appropriate stopping conditions, since from iteration 150 to iteration 350 , each iteration only causes a few pixel changes. Therefore a too relaxed stopping term will not able to stop the iterations while a strict stopping condition will stop the iteration before it is correctly segmented. The images here are obtained from CT head volumetric data set of Stanford. Figs. 3(d) to (f) show those iterations when different size and position of the initial curve are used. To segment the same image, the iteration used varies a lot when different positions and sizes of 
initial curves are used. It becomes even worse when complicated structure encountered. Fig. 4 shows the comparison between the level set and SVM based segmentation. As shown in Fig.4(c) the boundary find by SVM is not very accurate. Comparison of Fig. 3(c) and Fig. 4(d) shows that SVM is able to greatly accelerate level set segmentation. Previously 350 iterations are needed while 15 iterations are sufficient now. Figs. 5 and 6 show another two volumetric segmentation results. Fig. 7 gives results of coupled level sets and confidence map (fig. 7(d)). The brightness in the image indicates the confidence of the segmentation. The brighter the data, the more confident the segmentation is. Confidence map shows, as expected, that the boundary parts have lower confidence.
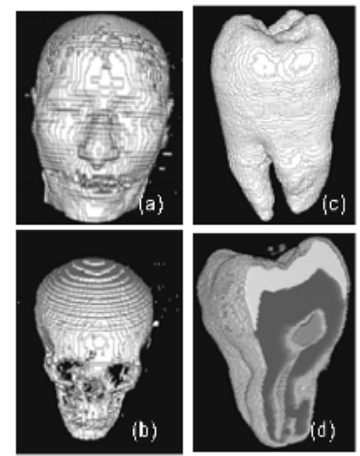

Fig. 6. Volumetric segmentation of another CT head and tooth. (a) The external part of the head. (b) Internal part of the head. (c) External part of tooth. (d) Internal part of the tooth.
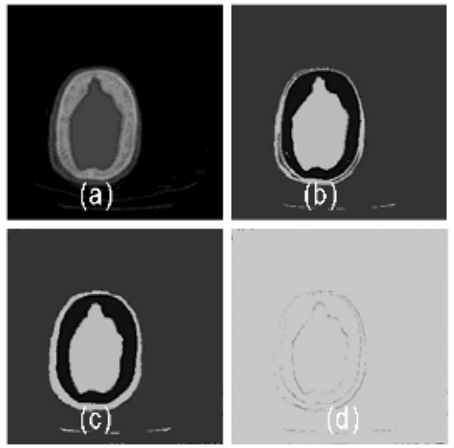

Fig. 7. Coupled level sets. (a) Original image. (b) Iteration 0. (c) Iteration 20. (d) Confidence map calculated.

\section{Conclusion}

In this paper, we first propose a Bayesian model to analyze the error in hierarchal level set and coupled level sets. The error analysis shows that coupled level sets are able to converge to more accurate boundaries even thought it takes much longer time to converge. Based on that analysis, an image processing approach adapted to the clinical setting is proposed and implemented. With the aid of techniques from the field of pattern recognition, we naturally combined hierarchical with coupled level set to achieve a fast and robust autonomous image segmentation framework for clinical initialization where the complicated setting for level sets can be transparent to user. The framework is not only able to achieve fast segmentation, but is also able to give more accurate boundary. To help the clinical physician to evaluate the segmentation results, a confidence map is computed. Two dimensional and volumetric images were used to test the framework. The experimental results are very promising. This framework works efficiently in a clinical setting where a specialist works with limited types of medical images. The classifiers need to be trained only once with representative images or slices segmented by hierarchical level set. The clinical physician just needs to indicate the classifier the image type. As a continuation of the work in this paper, 
we are currently implementing a real time segmentation system for selected types of clinical images.

\section{References}

1. V. Casseles, R. Kimmel, and G. Sapiro. Geodesic active contours. International Journal of Computer Vision, 22(1):61-79, 1997.

2. T. Chan and L. Vese. Active contour model without edges. IEEE Trans. Image Processing, vol. 24, 10(2):266-277, 2001.

3. C.-C. Chang and C.-J. Lin. Training nu-support vector classifiers: theory and algorithms. Neural Computation 13(9):2119-2147, 2001.

4. T. Chen and D. N. Metaxas. Gibbs prior models, marching cubes, and deformable models: a hybrid framework for 3D medical image segmentation, Proc. MICCAI, 703-710. 2003.

5. T. Deschamps. Curve and shape extraction with minimal path and level set techniques. Applications to 3D medical imaging. PHD thesis. University of Paris Daphine, 2001.

6. M. Holtzman-Gazit, D. Goldsher, and R. Kimmel. Hierarchical segmentation of thin structure in volumetric medical images. In Proc. MICCAI, 562-569, 2003.

7. R. Kimmel and A. M. Bruckstein. Regularized laplacian zero crossings as optimal edge integrators. International Journal of Computer Vision, 53(3):225-243, 2003.

8. A.E. Lefohn, J.E. Cates, and R.T. Whitaker. Interactive, GPU-Based Level Sets for 3D Segmentation, in Proc. MICCAI, 564-572. 2003.

9. S. Li, T. Fevens, and A. Krzyżak. An SVM based framework for autonomous volumetric medical image segmentation using hierarchical and coupled level sets. Proc. of Computer Aided Radiology and Surgery(CARS), 2004.

10. L. M. Lorigo, O. Faugeras, W. Grimson, R. Keriven, R. Kikinis, C.F. Westin, and Ayra Nabavi. Codimention-two geodesic active contours for the segmentation of tubular structures. Proc. of Computer vision and pattern recognition, 2000.

11. S. Osher and J. Sethian. Fronts propagating with curvature-dependent speed: algorithms based on Hamiltons-Jacobi formulations. J. Comput. Phys., 79(1):12-49, 1988.

12. C. Samson, L. Blanc-Feraud, G. Aubert, and J. Zerubia. A level set model for image classification. International Journal of Computer Vision, 40(3):187-197, 2000.

13. A. Tsai, A. Yezzi, and A. S. Willsky. Curve evolution implementation of the MumfordShah functional for image segmentation, denoising, interpolation, and magnification. IEEE Trans. Image Processing, 10:1169-1186, 2001.

14. A. Vasilevskiy and K. Siddiqi. Flux maximizing geometric flow. IEEE Trans. Pattern Anal. Machine Intel. , 24(12):1565-1578, December 2002.

15. L. Vese and T. Chan. A Multiphase Level Set Framework for Image Segmentation Using the Mumford and Shah Model. Intern. Journal of Computer Vision, 50(3):271-293, 2002.

16. X. Zeng, L. H. Staib, R. T. Schultz, and J. S. Duncan. Segmentation and measurement of the cortex from 3-D MR images using coupled surfaces propagation. IEEE Trans. Med. Imag., 18: 927-937, Sept. 1999.

17. H. K. Zhao, T. Chan, B. Merriman, and S. Osher. A variational level set approach to multiphase motion. J. Comput. Phys., 127:179-195. 1996.

18. S. Zhu and A. Yuille. Region competition: Unifying snakes, region growing, energy/Bayes/MDL for multi-band image segmentation. IEEE Trans. Pattern Anal. Machine Intel., 19(9): 884-900, 1996. 